\title{
Diagnostic and Therapeutic Approach to Sleep Disorders, High Blood Pressure and Cardiovascular Diseases: A Consensus Document by the Italian Society of Hypertension (SIIA)
}

\author{
Rita Del Pinto ${ }^{1}$. Guido Grassi ${ }^{2}$. Claudio Ferri ${ }^{2} \cdot$ Martino F. Pengo $^{3}$ - Carolina Lombardi ${ }^{3}$. Giacomo Pucci ${ }^{4}$. \\ Massimo Salvetti $i^{5}$ Gianfranco Parati $i^{3}$. on behalf of the Italian Society of Hypertension (SIIA) - SIIA Young \\ Researchers Study Group · President of SIIA - Past President of SIIA - Italian Society of Hypertension (SIIA)
}

Received: 28 December 2020 / Accepted: 6 February 2021

(c) The Author(s) 2021

\begin{abstract}
Hypertension is a major contributor to fatal/nonfatal cardiovascular diseases, and timely identification and appropriate management of factors affecting hypertension and its control are mandatory public health issues. By inducing neurohormonal alterations and metabolic impairment, sleep disorders have an impact on a variety of cardiovascular risk factors, including hypertension, and ultimately increase the risk of cardiovascular events. There is evidence that qualitative and quantitative sleep disorders are associated with resistant hypertension and with impaired circadian blood pressure variations. However, sleep disturbances are often unrecognized, or heterogeneity exists in their management by non-specialists in the field. This document by the Italian Society of Hypertension summarizes the updated evidence linking sleep disorders to hypertension and cardiovascular diseases, the major underlying mechanisms, and the possible management strategies. A simplified, evidence-based diagnostic and therapeutic algorithm for comorbid hypertension and common sleep disorders, namely obstructive sleep apnoea and insomnia, is proposed.
\end{abstract}

Keywords Sleep disorders $\cdot$ Blood pressure $\cdot$ Hypertension $\cdot$ Cardiovascular risk

The members of the SIIA Young Researchers Study Group, President of SIIA, Past President of SIIA, Italian Society of Hypertension (SIIA) listed in acknowledgement section.

Guido Grassi

guido.grassi@unimib.it

1 Division of Internal Medicine and Nephrology, Department of Life, Health and Environmental Sciences (MeSVA), Center for Hypertension and Cardiovascular Prevention, University of L'Aquila, San Salvatore Hospital, L'Aquila, Italy

2 Department of Medicine and Surgery, Milano Bicocca University, San Gerardo dei Tintori Hospital, Milan, Monza, Italy

3 Department of Medicine and Surgery, San Luca Hospital Scientific Institute, Institute for Research, Hospitalisation and Healthcare (IRCCS) Istituto Auxologico Italiano, Milano Bicocca University, Milan, Italy

4 Internal Medicine Division, Department of Medicine, University of Perugia, "Santa Maria” Hospital, Terni, Italy

5 Department of Clinical and Experimental Sciences, University of Brescia and General Medical Division 2, ASST Spedali Civili Hospital, University of Brescia, Brescia, Italy

\section{Introduction}

A proper sleep-wake cycle markedly influences a multitude of neuroendocrine systems, all of which are deeply involved in cardiovascular and metabolic homeostasis [1]. Accordingly, qualitative and/or quantitative sleep disorders adversely affect the physiology of the hypothalamic-pituitary-adrenal axis, the glycolipid metabolism and the cardiovascular system, thus exerting a considerable impact on the overall cardiometabolic risk [2].

Specifically, observational studies and experimental evidence fully support the existence of a close link between sleep disorders and arterial hypertension, cardiovascular disease, diabetes mellitus and/or obesity [3]. Because of the burden that all these diseases represent for the national health system, the risks of workplace accidents and traffic collisions and, finally, the losses due to reduced productivity, sleep disorders and excessive daytime sleepiness exert a relevant economic and social impact that fully justifies their inclusion among public health issues. In this context, adequate knowledge of sleep disorders and related solutions 
is essential for the clinician involved in the prevention and treatment of cardiovascular and metabolic diseases.

Among sleep disorders affecting blood pressure (BP), obstructive sleep apnoea (OSA) has been consistently associated with increased risk of incident hypertension and treatment-resistant hypertension, as well as impaired nocturnal physiological BP reduction ("dipping"), and is now recognised as one of the modifiable aggravating factors of the global cardiovascular risk [4]. In fact, OSA events are accompanied by acute changes in cardiovascular parameters, such as large fluctuations in BP and heart rate, associated with long-term changes in fluid homeostasis, all of which increase the risk of fatal and non-fatal cardiovascular events [5]. Delaying sleep onset also increases the likelihood of hypertension, with an estimated risk of $300 \%$ (odds ratio 3.27 ; $95 \%$ confidence interval 1.20-8.96). In women, sleep deprivation might represent a peculiar cause of vulnerability to hypertension and cardiovascular events [6, 7]. In addition, the expression of more than 700 genes involved in multiple functions, including metabolic control and redox status, is modified by sleep deprivation $[4,8]$. In parallel, a U-shaped association between sleep duration and all-cause mortality as well as increased cardiovascular risk has been described [9-11].

On these bases, hypertension guidelines emphasise the importance of investigating the possible presence of sleep disorders in patients with resistant arterial hypertension and loss of physiological nocturnal BP dipping, as well as the need for a multidimensional approach of the problem that includes both lifestyle changes (diet, exercise, weight loss) and medications [4].

He triad of "correct and timely diagnosis"- "lifestyle changes"- "resetting of the correct sleep-wake cycle" is gradually becoming the pillar of a correct approach to the issue of sleep disorders in cardiovascular prevention. Thus, accurately collecting a history of possible sleep disorders is essential in the evaluation of the patient with hypertension and/or increased cardiovascular and/or metabolic risk [4]. Lifestyle interventions, including diet and exercise, are especially relevant when excess weight is believed to favour a disordered sleep [12-15]. Restoring the correct function of the melatonin system-e.g. through oral administration of prolonged release melatonin at adequate dosages - might contribute to sleep restoration and cardiometabolic protection. Melatonin, in fact, is a hormone whose low levels have been frequently associated with the development of arterial hypertension [16], and whose administration in insomnia is also indicated in adults with cardiovascular comorbidities [17].

The document that follows this brief premise is promoted by the Italian Society of Hypertension in order to provide a summary of the state of the art of cardiovascular and metabolic implications of sleep disorders. The purpose of the document is to generate an updated and shared training and information tool, with the aim of standardising the approach of clinicians to the problem and optimising the multidimensional management of the hypertensive patient.

\section{Physiology and Pathology of Sleep}

As Heraclitus of Ephesus wrote: "To those who are awake, there is one world in common, but of those who are asleep, each is withdrawn to a private world of his own.", implying that the concept of sleep is an active process and is unique to each individual. Sleep is one of the most important behavioural characteristics of humans and animals, which forces them to spend a significant proportion of life in a state where reactions to environmental stimuli are inhibited and during which important cognitive processes are developed.

Although there are still many aspects to be defined, in particular concerning the meaning and function of sleep, research in the neurophysiology field over the last 30 years has made it possible to clarify some fundamental concepts such as the sleep-wake cycle and circadian rhythms.

As anticipated, sleep is a reversible state of interruption of motor interactions with the environment and is present in many animal species, even in simple organisms such as yeast. It is divided into rapid eye movement (REM) sleep, contrasted with non-REM sleep, where this oculographic feature does not occur.

This descriptive definition is associated with the presence/absence of a series of physiological functions that characterise REM and non-REM sleep. In the former, in addition to the known eye movements, there is a marked muscular atony of the antigravity muscles which spares the diaphragm. Non-REM sleep, which accounts for about $80 \%$ of total sleep time, is further divided into 4 categories, according to the first classification by Rechtschaffen and Kales, reduced to 3 (N1, N2 and N3) in 2007 after a re-evaluation by the American Academy of Sleep Medicine [18, 19]. During non-REM sleep, the brain works in a low-consumption mode that is visible at the electroencephalographic level through slow and broad waves; heart rate, respiratory rate and BP decrease in absolute values and become less variable.

The alternation of REM and non-REM sleep occurs several times (on average 4-5) during the night at intervals of about $90-120 \mathrm{~min}$. Stage N3 of non-REM sleep is more prominent in the first part of sleep, while REM sleep is more pronounced in the second part.

The alternation of sleeping and waking is part of a more complex process called a circadian rhythm. The circadian rhythm is a broader concept that is defined with a fundamental characteristic: it is an endogenous process that can occur even independently from the variation of the environmental features. However, at the same time, it is a process that is 
able to adapt to environmental stimuli while maintaining its own periodicity.

The ability of a cell or complex organism to keep track of time is inherent in a series of molecular mechanisms, the discovery of which earned Jeffrey C. Hall, Michael Rosbash and Michael W. Young the Nobel Prize in 2017 thanks to a series of studies on Drosophila melanogaster.

The presence of such molecular mechanisms that can establish the circadian rhythm universally in the different cells and tissues of an organism poses a risk of lack of synchronisation.

This risk is minimised by the presence of a hierarchical organisation of cells capable of maintaining a circadian rhythm, at the top of which is the suprachiasmatic nucleus $(\mathrm{SCN})$ in the hypothalamus. The SCN is located in a strategic area which is near the optic chiasm.

In this way it can receive optical stimuli from one of the most important external factors (Zeitgeber, from the German, "time giver"), able to synchronise a biological clock, i.e. light.

The extraordinary ability of the SCN to coordinate the biological clock of an organism has been confirmed in experiments where, by dispersing the single $\mathrm{SCN}$ neurons in culture, the molecular rhythms of each neuron were observed which, however, were out of step with one another [20].

In the intact SCN nucleus, on the other hand, a precise coordination of the various neurons is observed through the synaptic terminations, which use various neurotransmitters, including gamma-aminobutyric acid (GABA), allowing a common and synchronised output.

The activity of the SCN nucleus characterises a wide variety of cells and tissues of our body, starting from the paraventricular nucleus (PVN) of the hypothalamus on which it exerts an inhibitory action by conditioning the activity of the endocrine system and the autonomic nervous system with particular regard to the production of melatonin. Therefore, since the activity of SCN is dependent on light stimuli, an increase in SCN activity during exposure to light is effectively able to reduce the production of melatonin by the pineal gland [21].

Melatonin is a fundamental neurohormone for the synchronisation of the biological clock. It is produced by the pineal gland during the night hours reaching maximum concentrations in the blood between 2:00 a.m. and 4:00 a.m. and then gradually decreasing as the morning approaches.

Neurones located in the brain stem are also involved in the circadian regulation of sympathetic and vagal activity and the relative modulation by the autonomic nervous system of the cardiovascular system between day and night [22].

Sleep disorders can be grouped according to different classifications, one of the most widely used is the International Classification of Sleep Disorders (ICSD), which is now in its third edition and produced by the American Academy of Sleep Medicine [23].

This classification divides sleep disorders into the following categories:

1) Insomnia

2) Sleep-related breathing disorders

3) Central disorders of hypersomnolence

4) Circadian rhythm sleep-wake disorders

5) Parasomnias

6) Sleep-related movement disorders.

The first group includes insomnia, which is defined as a disorder characterised by difficulty in falling asleep or staying asleep, or by nonrestorative sleep. This disorder affects the subjective feeling of well-being, even causing daytime psychic, cognitive and somatic alterations.

This is the most frequently reported sleep disorder in clinical practice and in the general population. It is frequently associated with other disorders; in particular, the most well-known and described link in the scientific literature is undoubtedly with mental disorders. In particular, it has been shown that the presence of insomnia is capable of predicting the onset of a depressive episode, anxiety, alcohol abuse, or psychosis and represents a risk for the development of suicidal ideation and behaviour [24].

Insomnia is also associated with high cardiovascular morbidity and mortality, particularly when accompanied by a short sleep duration (Fig. 1) [25]. One possible explanation of this could be the altered BP profile over $24 \mathrm{~h}$ of sleepless patients with reduced dipping and nocturnal hypertension [26].

The second group encompasses all respiratory disorders that occur during sleep, the most prevalent being OSA syndrome (OSAS). OSAS is determined by a transient but repeated interruption of breathing during sleep. This phenomenon causes a qualitative change in night-time rest, which may lead to symptoms during waking, such as excessive daytime sleepiness and fatigue.

As previously seen, sleep induces a state of general muscle relaxation, particularly in the REM stage. The loss of muscle tone can cause the upper airway walls (hypopharynx) to narrow and begin to vibrate, thus producing the wellknown snoring phenomenon. In extreme conditions, an actual collapse of the walls can occur, or even the complete airway obstruction. In this case our brain briefly interrupts sleep by awakening; this leads to the recovery of adequate muscle tone, stopping the apnoea phenomenon. Patients suffering from OSA, however, may not be aware of the many awakenings associated with apnoea, because sometimes wakefulness and lighter sleep are not so intense or prolonged that the individual reaches a state of consciousness. At morning awakening, however, there may often be a feeling 
Fig. 1. Major mechanisms of the association between sleep disorders with increased cardiovascular morbidity and mortality. See text for details. $O S A$ obstructive sleep apnoea

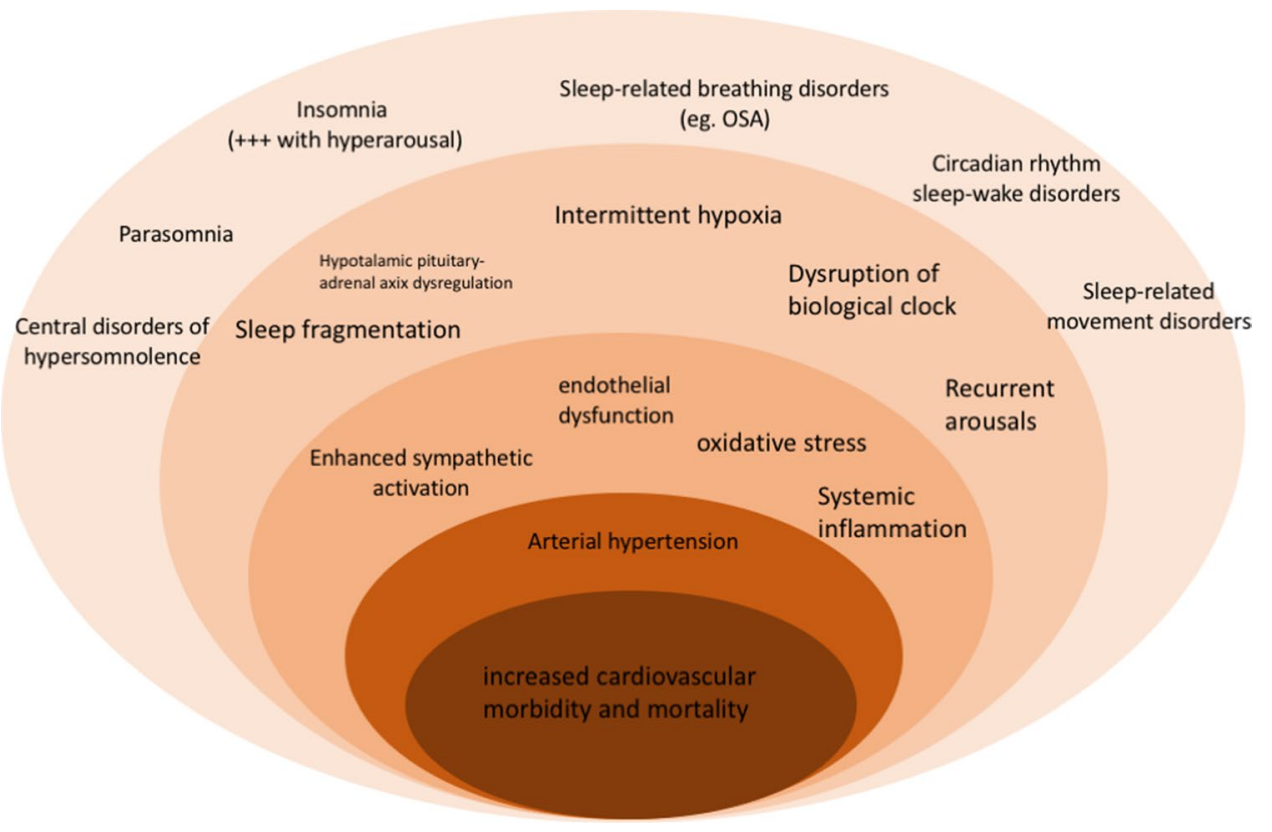

of not having had a restful night's sleep and symptoms such as excessive sleepiness, fatigue or headache-characteristic expressions of poor sleep quality—may also appear.

Numerous studies have demonstrated the association between hypertension and OSAS both from a pathophysiological point of view, documenting transient pressure rises due to an increase in sympathetic tone during obstructive events, and from an epidemiological point of view (Fig. 1) [27].

In addition to obstructive apnoea, central apnoea is also frequently found in patients with cardiovascular diseases such as heart failure. During central apnoea there is no obstruction to the upper airways; instead, pauses in breathing occur due to lack of inspiratory chest or abdominal movements. The causes of this disorder are manifold and may be found in the mechanism of respiratory control in the central nervous system (CNS). Respiratory stimulation to the major muscles involved in breathing, such as the diaphragm, depends on several stimuli, including nerve afferents originating in chemoreceptors. Unstable breathing that predisposes to hyperventilation and drops in $\mathrm{PaCO}_{2}$ below the apnoeic threshold results in a reduction in the output to the respiratory muscles and therefore in central apnoea.

Many of the respiratory sleep disorders result in daytime hypersomnia due to sleep disturbed by apnoea and other respiratory events. There are other sleep disorders that are characterised by daytime hypersomnia not secondary to significant functional sleep disruptions.

This is the case with primary hypersomnias, which include narcolepsy - a pathology characterised by excessive daytime sleepiness, often experienced as recurrent involuntary sleep attacks, which occur during the day. It is caused by an inability of the brain to regulate the sleep-wake cycle in a physiological manner. In patients with narcolepsy, there is a rapid and atypical emergence of REM sleep, in the 15-20 min that elapse after falling asleep, and an intrusion of REM sleep during the day. Narcolepsy affects on average 1 in 2000 people $(0.05 \%$ of the population) and is characterised, in addition to daytime sleepiness, by three key symptoms caused by the intrusion of REM sleep during wakefulness: cataplexy, a sudden, brief, reversible episode of muscle weakness that occurs in conjunction with emotional stimuli; sleep paralysis, an experience that occurs while sleeping, during which the subject suddenly feels unable to move or speak and hypnagogic or hypnopompic hallucinationsvivid dreamlike experiences that occur on falling asleep or waking from sleep respectively.

The ICSD classification also includes circadian rhythm disorders that result from a lack of synchronisation between sleep-wake cycles and the schedules imposed by social needs, resulting in fatigue, poor work and school performance and sleep disorders, particularly difficulty in falling asleep or waking up at the desired times.

Circadian rhythm disorders include: delayed sleep phase syndrome, in which subjects tend to fall asleep between 3:00 a.m. and 6:00 a.m. and wake up between 12:00 p.m. and 3:00 p.m.; advanced sleep phase disorder-in these subjects there is a tendency to fall asleep and wake up early compared to the conventional setting; jet-lag disorder, in subjects who travel across at least two different time zones; and shift work sleep disorder, frequent in workers with shifts that alternate between day and night.

Parasomnias represent a broad and heterogeneous group of sleep disorders that consist of anomalous and involuntary 
behaviours during sleep, sometimes aimed at achieving a goal.

They are further divided into NREM sleep parasomnias, such as sleepwalking and night terrors, and REM sleep parasomnias, such as REM sleep behaviour disorder.

The latter is characterised by the loss of physiological muscle paralysis during REM sleep. Therefore, during the episodes, patients present excessive motor activity, often characterised by abrupt behaviours (such as screaming, punching and kicking), in relation to the content of their dreams.

This disease can be associated in $40 \%$ of cases with some neurodegenerative diseases, such as Parkinson's disease, multisystem atrophy and some forms of dementia.

Finally, sleep movement disorders are characterised by the appearance of simple and repetitive movements that disturb sleep and its onset. Restless legs syndrome (RLS) is common, and manifests itself with a feeling of restlessness in the lower limbs at rest, typically in the evening, which induces the subject to move his or her legs and to walk, compromising falling asleep and the continuity of sleep. It often comes with rhythmic movements of the limbs during sleep, which disturb sleep and are associated with microawakenings as well as transient increases in heart rate and BP during sleep.

Given the impact of periodic movements to the lower limbs on night-time BP [28, 29], RLS has been associated with the development of cardiovascular diseases (Fig. 1) [28]. However, this report needs confirmation from subsequent longitudinal studies.

\section{Sleep Disorders and Cardiovascular Diseases}

For many years, the role of sleep in human pathophysiology has been the subject of attention almost exclusively by psychologists and neuroscience experts, but in more recent times the concept of sleep as a restorative process in the wider sense has become more established, extending to the physiological functions of the whole body. The link between abnormal sleep duration and diseases had already been hypothesised by Hippocrates, who had observed that "Both sleep and insomnolency, when immoderate, are bad". The pace of modern life has caused a significant change in sleep duration and quality compared to the past, with an increase in the proportion of people claiming to sleep less than $7 \mathrm{~h}$ a day in recent years (from 22\% observed in 1977 to $29 \%$ in 2009) [30, 31]. In particular insomnia, which is the most prevalent sleep disorder, seen in about a third of the people in the general population in Europe [30], has been associated with a number of pathological conditions. Over time, evidence has accumulated to support a significant relationship between sleep disorders and metabolic and cardiovascular diseases (Fig. 1) [31].

\subsection{Sleep Duration and Cardiovascular Risk Factors}

A short sleep duration (SSD), defined as $<6$ or $7 \mathrm{~h}$ a day of sleep, is associated with a higher prevalence of obesity. The results of some longitudinal studies also seem to support a relationship between SSD and incident obesity, with an increase in risk of 55\%; the correlation seems particularly evident when the duration of sleep falls below $4 \mathrm{~h}$ [31]. Case studies indicate that people with SSDs tend to have more snacks between meals, have more irregular eating habits, consume more calories and eat more fat. Even those who sleep too much, or "long sleepers" (LS), are more often obese and tend to consume more calories and less healthy food. The relationship between abnormalities in sleep duration and energy consumption is a more complex and less researched area, although the role of an alteration in the relationship between energy intake and calorie consumption in both SSD and LS subjects seems evident [31].

Abnormalities in sleep duration are also associated with a higher prevalence of diabetes mellitus. A meta-analysis that included more than 10,000 subjects showed a significant risk of developing diabetes in those who sleep less than 5-6 h or more than $8-9 \mathrm{~h}$ per night, with a relative risk of developing diabetes of 1.28 and 1.48 , respectively, during an average follow-up of approximately 10 years [32].

Some studies have also reported a relationship between short sleep duration and arterial hypertension, but the data are quite heterogeneous. The most recent meta-analysis [33] appears to indicate a U-shaped relationship in crosssectional studies, with an increased risk of hypertension for both those with reduced sleep duration and those with increased sleep duration. In longitudinal studies, however, there is a significant relationship with arterial hypertension for subjects with short sleep duration but not for LS [33]. This relationship seems to be more evident in younger ages: a recent analysis of the available data showed an increase in the risk of arterial hypertension of $51 \%$ among adolescents with short sleep duration, in the absence of significant associations with long sleep duration [34].

\subsection{Sleep Duration and Overt Cardiovascular Disease}

The association described so far between sleep duration and obesity, metabolic abnormalities and arterial hypertension is also a valid prerequisite for a relationship with overt cardiovascular diseases, which has been described in various studies, although with a slight heterogeneity in the reported results. Among the most recent, a cross-sectional analysis carried out on 32,152 subjects within the National Health 
and Nutrition examination Survey (NHANES) [35] showed a significant association between sleep duration and cardiovascular diseases, and in particular, in patients with short sleep duration, a higher prevalence of subjects with a clinical history of cerebral stroke (odds ratio, OR 1.45; CI 95\% 1.23-1.70), and heart failure (OR 1.65; CI 95\% 1.40-1.95) was observed, even after correction for several possible confounding factors. A long sleep duration was also associated with cardiovascular disease, and in particular with stroke (or 1.81; CI 95\% 1.37-2.34) and heart failure (OR 1.47; CI 95\% 1.08-1.97). The interpretation of the results of part of the available studies is limited by their cross-sectional nature, which does not allow a certain causal attribution between sleep duration and observed events. For longitudinal studies, a broad meta-analysis [36] including 15 studies and 474,684 subjects analysed the relationship between sleep duration and long-term risk of cardiovascular events, with a followup duration of 6.9-25 years). As regards the risk of stroke, the authors described an increased risk of stroke (fatal and non-fatal) in subjects with short sleep duration (relative risk, RR 1.48, CI 95\% 1.22-1.80, $\mathrm{p}=0.0001$ ) and also in subjects with long sleep duration (RR 1.65, CI 95\% 1.45-1.87, $\mathrm{p}=0.0001$ ).

Similar conclusions were also found regarding the risk of coronary events (fatal and non-fatal), with a relative risk of 1.48 (CI 95\% 1.22-1.80, p = 0.0001) for subjects with short sleep duration and 1.38 (CI 95\% 1.15-1.66, $\mathrm{p}=0.0005$ ) for those who reported long sleep duration. The meta-analysis showed inconclusive results with regard to total cardiovascular events and cardiovascular mortality; this finding may in part be linked to a less precise definition of the events on which the analysis is based, but it could also be justified by the presence of confounding factors not considered in the multivariate model adopted in this study and which could have influenced other similar studies in literature as well. The analysis of data from the United Kingdom Biobank (500,000 healthy adult subjects) recently confirmed the increased risk of myocardial infarction in subjects with less than $6 \mathrm{~h}$ of sleep ( $20 \%$ increase in risk). It also showed a $34 \%$ increase in risk associated with sleeping longer than $9 \mathrm{~h}$ [37]. The results, in the same study, of the Mendelian randomisation analysis supported a causal link between short sleep duration and risk of acute myocardial infarction. Very little data is available regarding the relationship between sleep duration and cardiovascular events in patients with confirmed cardiovascular disease. Kim et al. recently analysed the relationship between sleep duration and risk of events in about three thousand patients with confirmed coronary artery disease included in the "Emory Cardiovascular Biobank" [9]. Short or long sleep duration has been associated with a significant increase in the risk of death from all causes during an average follow-up of 2.8 years. When the authors focused on mortality from cardiovascular causes, a significant relationship with short sleep duration but not with long sleep duration emerged, after correction for possible confounding factors.

As for the relationship between sleep duration and atrial fibrillation, the data available in the literature are not conclusive, although some, but not all, case studies seem to suggest a U-shaped relationship, as for cerebrovascular and coronary events $[38,39]$.

In conclusion, epidemiological data, although heterogeneous, convincingly supports the presence of a relationship between short sleep duration and risk of cardiovascular events (Table 1); this relationship is also reasonably supported by the possible role of hormonal alterations associated with a reduced sleep duration (possible increase in cortisol levels, suppression of leptin levels and increase in ghrelin, reduction of melatonin levels, alterations in the endocannabinoid system), insulin resistance, disruption in eating behaviour that can lead to being overweight and obesity, release of proinflammatory cytokines [31]. The relationship with long sleep duration and risk of cardiovascular complications seems to be less well established. Indeed, many confounding factors may play a significant role in this relationship. Prospective studies with strict standardisation, with analysis of all possible confounding factors and quantitative evaluation of sleep duration may better define the relationship between sleep duration and cardiovascular events.

Table 1 Relationship between sleep duration, determinants of cardiovascular risk, and cardiovascular events

\begin{tabular}{lll}
\hline & $\begin{array}{l}\text { Short sleep duration } \\
(<7 \mathrm{~h})\end{array}$ & $\begin{array}{l}\text { Long sleep } \\
\text { duration }(>9 \\
\mathrm{h})\end{array}$ \\
\hline Calories intake & $\uparrow$ & $\uparrow$ \\
Energy expenditure & $\downarrow ?$ & $?$ \\
Obesity & $\uparrow \uparrow$ & $\uparrow$ \\
Arterial hypertension & $\uparrow$ & $\uparrow ?$ \\
Diabetes mellitus & $\uparrow$ & $\uparrow \uparrow$ \\
Atrial fibrillation & $\uparrow ?$ & $\uparrow ?$ \\
Stroke & $\uparrow$ & $\uparrow \uparrow$ \\
Heart failure & $\uparrow \uparrow$ & $\uparrow$ \\
Myocardial infarction & $\uparrow$ & $\uparrow \uparrow$ \\
Coronary events & $\uparrow \uparrow$ & $\uparrow$ \\
All cause death & $\uparrow$ & $\uparrow$ \\
Cardiovascular death & $\uparrow$ & $=$ \\
\hline
\end{tabular}

Upwards/downwards arrows indicate increased/decreased risk for the relative condition, while the equal sign indicates similar risk compared with intermediate sleep duration. The same number of arrows indicates no difference in risk between short and long sleep duration. Question marks indicate controversial evidence. See text for details 


\subsection{Qualitative Sleep Abnormalities and Cardiovascular Risk}

Disturbances in the quality of sleep are a further element that, other than sleep duration, could exert a negative influence on the cardiovascular system (Fig. 1).

It is not easy to correctly classify the presence of qualitative sleep abnormalities. In fact, for a correct diagnosis, it is often needed to undergo complex investigations, such as polysomnography, which are difficult to apply as a screening method in the general population. Moreover, it is not always possible to discriminate to what extent qualitative sleep abnormalities cause unfavourable effects on the cardiovascular system regardless of their repercussions on the duration of the sleep itself.

Qualitative sleep disturbances may include frequent nocturnal awakenings which cause excessively fragmented sleep, respiratory sleep disorders, including OSA, and periodic movements of the limbs in sleep, a feature of restless leg syndrome (Table 2).

One aspect toward which the scientific interest is constantly growing is represented by circadian rhythm sleep disorders, which often affect shift workers who frequently switch between daytime and night-time shifts.
The presence of fragmented sleep, interrupted by frequent awakenings, regardless of their causes, has a negative effect on the cardiovascular system in that the structure of sleep, that is the temporal succession of the various phases of sleep, is altered. There is consistent scientific background attributing a protective significance to REM (rapid eye movement) sleep duration in relation to cardiovascular disease. This includes data from the Cardiovascular Health Study where it was observed a linear and inverse relationship between REM sleep duration and the risk of atrial fibrillation [40]. Sympathetic tone is high during REM sleep, suggesting that those with less REM sleep may be characterized by higher levels of vagal tone, which may favor AF appearance. However, lower odds of AF were reported among those with a longer duration of slow-wave sleep, which is typically characterized by greater cardiac vagal modulation. The cross-sectional nature of the available evidence, however, does not allow to clarify whether differences in sleep architecture were an effect (rather than a cause) of AF. Among the mechanisms of damaging effect, an increase in sympathetic tone and a decrease in parasympathetic tone have been identified as factors that are also associated with high levels of nocturnal BP and reduced nocturnal BP drop. Although

Table 2 Qualitative sleep disorders and cardiovascular risk

\begin{tabular}{|c|c|c|c|}
\hline Definition & Description & $\begin{array}{l}\text { Association with car- } \\
\text { diovascular risk factor }\end{array}$ & $\begin{array}{l}\text { Association with } \\
\text { cardiovascular } \\
\text { events }\end{array}$ \\
\hline Insomnia & $\begin{array}{l}\text { Quantitative and qualitative sleep disorder characterized by } \\
\text { difficulty falling asleep, frequent nocturnal interruption, early } \\
\text { awakening, accompanied by daytime symptoms related to this } \\
\text { condition }\end{array}$ & $\uparrow \uparrow$ & $\uparrow \uparrow$ \\
\hline Fragmented sleep & $\begin{array}{l}\text { Qualitative alteration of sleep characterized by frequent and brief } \\
\text { awakenings }\end{array}$ & $\uparrow=$ & $\uparrow \uparrow$ \\
\hline Sleep disordered breathing & $\begin{array}{l}\text { A group of clinical conditions characterized by abnormal breath- } \\
\text { ing during sleep. They include: obstructive apnoeas, central } \\
\text { apnoeas, hypoventilation and sleep-related hypoxemia }\end{array}$ & $\uparrow \uparrow$ & $\uparrow \uparrow$ \\
\hline Obstructive sleep apnoea & $\begin{array}{l}\text { Episodes of partial or complete closure of the upper airways dur- } \\
\text { ing sleep leading to respiratory interruption, desaturation and } \\
\text { awakening. If accompanied by daytime symptoms, it is called } \\
\text { "obstructive sleep apnoea syndrome" }\end{array}$ & $\uparrow \uparrow \uparrow \uparrow$ & $\uparrow \uparrow \uparrow$ \\
\hline Periodic limb movements in sleep & $\begin{array}{l}\text { Repetitive contraction of the upper or lower limbs during sleep, } \\
\text { which often lead to frequent awakenings and fragmented sleep }\end{array}$ & $=$ & $=$ \\
\hline Restless leg syndrome & $\begin{array}{l}\text { Motor restlessness, uncontrolled movements and contractions } \\
\text { of the lower limbs that occurs in the phases of falling asleep, } \\
\text { sleep and awakening. When conscious, the patient feels a } \\
\text { sensation of discomfort in the legs, accompanied by the need } \\
\text { to move them continuously }\end{array}$ & $\uparrow \uparrow$ & $\uparrow$ \\
\hline Circadian rhythm sleep disorders & $\begin{array}{l}\text { Alterations caused by the desynchronization between the endog- } \\
\text { enous sleep-wake rhythms and the normal external light-dark } \\
\text { cycle, from endogenous causes (delayed or advanced sleep } \\
\text { phases) or exogenous (intermittent night shifts) }\end{array}$ & $\uparrow \uparrow \uparrow$ & $\uparrow \uparrow$ \\
\hline
\end{tabular}

The arrows and the equal sign indicate the strength of the association (equal sign: no association; one, two, three, or four arrows: mild, moderate, strong, very strong association). See text for details 
these are still preliminary observations, the results of some studies which have suggested a link between fragmented sleep, regardless of total duration, and the risk of developing arterial hypertension are important in this context [41].

Obstructive sleep apnoea (OSA), a disease affecting about $49 \%$ of men and $23 \%$ of women living in Europe [42], is by far the most studied category of sleep disorders in relation to cardiovascular disease. Large-scale studies suggested a dosedependent link between the number of OSAs and the risk of developing arterial hypertension, which also increases for mild forms (between 5 and 15 apnoea-hypopnoea events per hour) [43]. In this context, there are interesting data supporting a link between OSA, atherogenic dyslipidaemia [44] and the onset of metabolic syndrome [45].

One of the main mechanisms that affect the high cardiovascular risk in OSA is the so-called "hypoxic load"-a combined index of the frequency and extent of the episodes of oxygen desaturation during sleep, able to act as a trigger of endothelial dysfunction and systemic inflammation [46].

The potential causal link between OSA and cardiovascular events is supported by the results of prospective longitudinal studies. In the Sleep heart Study, in subjects under 70 years of age, OSA was an independent predictor of the 5 -year incidence of coronary events (HR 1.10), ischaemic stroke (HR 1.58) and congestive heart failure (HR 2.38) [47]. The potential protective effect of treating OSA in terms of reducing cardiovascular risk is currently unclear.

Several screening tools exist to aid in identification of OSA patients, with five being fairly easy to administer: Stop, STOP-BANG (SB) (Fig. 2, Box 1), Epworth Sleepiness Scale (ESS) (Fig. 2, Box 2), the Berlin Questionnaire (BQ), and the 4-Variable screening tool (4-V) (Fig. 2, Box 3) [48-52]. The ESS is a simple and validated questionnaire for assessing subjective daytime sleepiness or sleep propensity in adults in the context of sleep disorders [50]. The BQ consists of 3 categories (snoring and apnoea frequency, daytime symptoms, and BP) related to the risk of having OSA, with individuals being at high risk if there are 2 or more categories where the score is positive, and low risk if there is only 1 or no categories where the score is positive [51]. The 4-V is an equation consisting of four variables including gender, BMI, BP, and self-reported snoring [52]. SB has the highest sensitivity (97.6\%), followed by the BQ $(87 \%)$, while the $4-\mathrm{V} \geq 14$ has the highest specificity ( $74.4 \%$ ), followed by the ESS (67\%). Recently, a weighted ESS scoring system was developed to improve the accuracy of the AHI prediction, resulting in strong capability in predicting the patients without OSAS or with severe OSAS and improvement in screening the patients with simple snoring [53]. However, external validity and generalizability of the weighted ESS need further investigations.
Periodic limb movements in sleep (PLMS) are often found during nocturnal apnoea-hypopnoea episodes in subjects with OSA. These abnormalities may also occur isolated (primary PLMS) or within the context of other diseases, such as peripheral neuropathies, nephropathy or psychiatric disorders (secondary PLMS). Subjects with PLMS typically have shorter phases of N3 sleep and REM sleep. Although this is sufficient to hypothesize a relationship between PLMS, arterial hypertension and cardiovascular risk, current epidemiological observations, in contrast to the initial observations, do not seem to support a relationship between PLMS and cardiovascular risk factors independently from the effect of the confounding factors [54].

However, in the analysis of the relationship between PLMS and cardiovascular risk, if the field of investigation is restricted to those with symptoms compatible with restless leg syndrome (RLS), characterised by the discomfort that appears in the legs during rest, it is possible to find evidence of a possible relationship between this condition and cardiovascular risk. In the Nurses' Health Study, in fact, subjects who reported symptoms compatible with RLS appeared to be at increased risk of developing myocardial infarction (OR 1.8) and death from ischaemic heart disease (OR 1.5) over time; this risk was even greater when considering subjects with duration of symptoms greater than at least 3 years [55]. In contrast, many other subsequent studies did not confirm this initial evidence, indicating the heterogeneity of the definition and classification of RLS, the methodologies adopted for diagnosis, and the concomitant impact on cardiovascular risk of conditions associated with secondary RLS such as diabetic neuropathy as possible explanations, which often were not adequately considered in the analysis of the results.

The category of qualitative sleep disorders includes the cardiovascular consequences related to shift working, a condition to which an ever-increasing number of workers are exposed. It is estimated that around $18 \%$ of workers in Europe carry out at least $25 \%$ of their work at night. Although some of the adverse effects on the cardiovascular system of shift workers are due to sleep deprivation, there are other potential mechanisms of harm that can be identified in the disturbance of the circadian rhythm of sleep and in the increased predisposition to unbalanced diets and unhealthy lifestyles [56].

In a meta-analysis that attempted to quantify the adverse effects of shift working on the cardiovascular system, it was observed that this condition increases the risk of cardiovascular events, particularly coronary events, by $17 \%$. Moreover, this risk would appear to increase cumulatively from the fifth year of shift working [57]. The potential impact of prolonged rest periods following night-time shift working and of the type of shift (clockwise, anti-clockwise) on quality of life, as well as on the cardiovascular risk associated 
Fig. 2. Major screening questionnaires for obstructive sleep apnoea. See text for details

Box 1.

\section{STOP-BANG Questionnaire}

Snoring: Do you snore loudly (louder than talking or loud enough to be heard through closed doors)? Tired: Do you often feel tired, fatigued, or sleepy during daytime?

Observed: Has anyone observed you stop breathing during your sleep?

Blood Pressure: Are you being treated for BP?

BMI: $>35 \mathrm{~kg} / \mathrm{m}^{2}$ ?

Age: $>50$ years old?

Neck circumference: $>40 \mathrm{~cm}$ (measured by staff)?

Gender: male?

One point awarded for each question answered with "Yes".

Scores $\geq 2 / 4$ for Stop or $\geq 3 / 8$ for STOP-BANG indicate high risk for OSA.

Highest sensitivity and lowest specificity across strata of OSA severity (mild, moderate, severe).

\section{Box 2.}

ESS

Situation

Sitting and reading

Watching TV

Sitting inactive in a public place (e.g., a theater or a meeting)

As a passenger in a car for an hour without a break

Lying down to rest in the afternoon when circumstances permit

Sitting and talking to someone

Sitting quietly after a lunch without alcohol

In a car, while stopped for a few minutes in traffic

Total scores

\begin{tabular}{cccc}
\multicolumn{4}{c}{ Chance of dozing } \\
$\boldsymbol{I}$ & $\boldsymbol{I I}$ & $\boldsymbol{I I I}$ & $\boldsymbol{I} \boldsymbol{V}$ \\
0 & 1 & 2 & 3 \\
0 & 1 & 2 & 3 \\
0 & 1 & 2 & 3 \\
0 & 1 & 2 & 3 \\
0 & 1 & 2 & 3 \\
0 & 1 & 2 & 3 \\
0 & 1 & 2 & 3 \\
0 & 1 & 2 & 3 \\
& $0-24$ &
\end{tabular}

I: would never doze. II, III, IV: slight, moderate, and high chance of dozing.

Scores $>10$ are considered as abnormal sleepiness, with better sensitivity and specificity in severe rather than mild ad moderate OSA.

Box 3.

The 4-V equation

$\mathrm{OSA}=($ gender $* 4)+(\mathrm{BMI}$ range $)+(\mathrm{BP}$ range $)+($ snoring $* 4)$

Gender (points): men (1); women (0).

BMI range (points): $<21$ (1); 21-22.9 (2); 23-24.9 (3); 25-26.9 (4); 27-29.9 (5); $\geq 30$ (6).

BP range (points): $\mathrm{SBP}<140$ or DBP $<90 \mathrm{mmHg}$ (1); SBP 140-159 or DBP 90-99 mmHg (2); SBP

160-179 or DBP $100-109 \mathrm{mmHg}(3)$; SBP $>180$ or DBP $>110 \mathrm{mmHg}$ (4).

Snoring (points): almost every day or often = "yes" (1); sometimes or almost never $=$ "no" $(0)$.

Scores $\geq 14$ have the highest specificity and PPV across strata of OSA severity (mild, moderate, severe). with shift working, represents a subject of great interest for study in the scientific landscape of today.

In conclusion, the data available in the literature, summarised in Table 2, support the existence of a significant relationship between qualitative sleep disturbances and confirmed cardiovascular disease.

\section{Treatment for Sleep Disorders}

In order to properly set up the treatment of sleep disorders, especially when associated with arterial hypertension or other cardiovascular risk factors, a correct hypnological diagnosis should be made based on proper gathering of medical history information, on physical examination and often on the performing of polysomnographic 
examination of the most suitable type for the specific suspected diagnostic.

In addition to the general collection of patient data and the pharmacological history, the medical history should also be aimed at identifying sleep habits (sleep and waking schedule, number of awakenings during the night, daytime sleeps, etc.), and the presence of specific symptoms for different sleep disorders (e.g. snoring, daytime sleepiness, morning headache, degree of feeling rested in the morning, involuntary movements in sleep, etc.).

The physical examination is mainly useful for identifying the signs associated with OSA, in particular obesity, mainly central, macroglossia, the conformation of the uvula, tonsils and palate, skeletal abnormalities of the upper and lower jaw, possible nasal obstruction which, although not a cause of the respiratory disorder, may complicate treatment management, in particular with positive pressure devices.

The cardiothoracic physical examination is essential for any signs of lung disease, baseline heart disease or skeletal spinal diseases such as kyphoscoliosis. For all non-respiratory sleep disorders, an in-depth neurological physical examination is also indicated.

Diagnostic investigations are usually required when specific symptoms and signs suggest the presence of a sleep apnoea, epilepsy with seizures during sleep, narcolepsy, periodic limb movements in sleep, or other disorders for which diagnosis is based on the identification of characteristic polysomnographic findings. In other cases (such as psychophysiological insomnia, bad sleep habits, transient stress, disrupted sleep from work shifts), polysomnographic investigations are not always necessary. In some cases, a video recording may be associated with polysomnographic monitoring and in this case the examination is performed in the sleep lab. In the vast majority of cases, however, the tests carried out at home with portable polysomnographs are sufficient to establish a correct diagnosis particularly in the context of respiratory sleep disorders.

The therapeutic setting for sleep disorders is therefore closely linked to the specific diagnosis performed by the sleep medicine specialist. In this context, we will discuss in detail the treatment of the two sleep disorders most closely related to cardiovascular diseases and arterial hypertension: insomnia and OSA, also discussing the impact of these treatments on BP levels.

\subsection{Treatment of OSA}

The treatment of choice for sleep respiratory disorders, in particular for OSA, is based essentially on the severity of the disorder, the general characteristics of the patient and the possible presence of comorbidities (Fig. 3).

Certainly, in the face of the presence of overweight/obesity, the first step is to obtain weight loss, aiming to modify the lifestyle with an increase in daily physical activity, in addition to dietary changes.

It is also useful to avoid excessive consumption of alcohol in the evening and sedative drugs that can aggravate the respiratory sleep disorder.

The gold standard of treatment of OSA is still the use of continuous positive airway pressure (CPAP) or bilevel positive airway pressure (BIPAP) devices through nasal or oronasal masks, depending on the shape of the patient's face.

These ventilation support devices provide positive airway pressure which allows the patency of the airways to be maintained during sleep. In order to be able to identify the specific therapeutic pressure for each patient capable of correcting OSA and snoring, it is necessary to titrate the pressure of the air delivered by the device by means of nocturnal adaptation polygraph.

In the case of positional sleep apnoea (i.e., occurring when the patient sleeps in supine decubitus) it may be useful to use supine position distractor devices, which essentially force the patient to adopt a lateral decubitus position during sleep.

In recent years, mandibular advancement devices (MAD) have been developed, which are worn during sleep and serve to fix the lower jaw slightly forward of the upper jaw, so that it does not tend to obstruct the airways during sleep.

There are also a number of surgical solutions for snoring and for apnoea, ranging from upper airway surgery to bariatric surgery in severely obese patients. The type of intervention in the upper airways must however be evaluated by specialists experienced in sleep medicine after fibroscopic and functional investigations, in order to establish the correct surgical indication [58].

\subsection{Treatment of Insomnia}

First-line treatment for insomnia is cognitive-behavioural therapy, which should ideally be performed before hypnotics are prescribed. Good sleep hygiene is one component of cognitive-behavioural therapy that is important whatever the cause and is often the only treatment that patients with mild problems need.

Cognitive-behavioural therapy for insomnia focuses on managing common thoughts, worries and behaviours that interfere with sleep (Fig. 4) [59].

General guidelines for the use of hypnotics aim to minimise abuse, misuse and dependence.

All hypnotics (except ramelteon, low doses of doxepin and suvorexant) act on the benzodiazepine recognition site on the $\gamma$-aminobutyric receptor (GABA) and increase the inhibitory effects of $\gamma$-aminobutyric acid. Melatonin, which in Italy is registered as a drug in its $2 \mathrm{mg}$ prolongedrelease formulation and has a specific first-line indication 
Fig. 3. Diagnostic workup and treatment options for obstructive sleep apnoea. See text for details. $O S A$ obstructive sleep apnoea, $B D Z$ benzodiazepines, $C P A P$ continuous positive airway pressure, $B I P A P$ bilevel positive airway pressure, $A S V$ adaptive servo ventilation, $M A D$ mandibular advancement devices

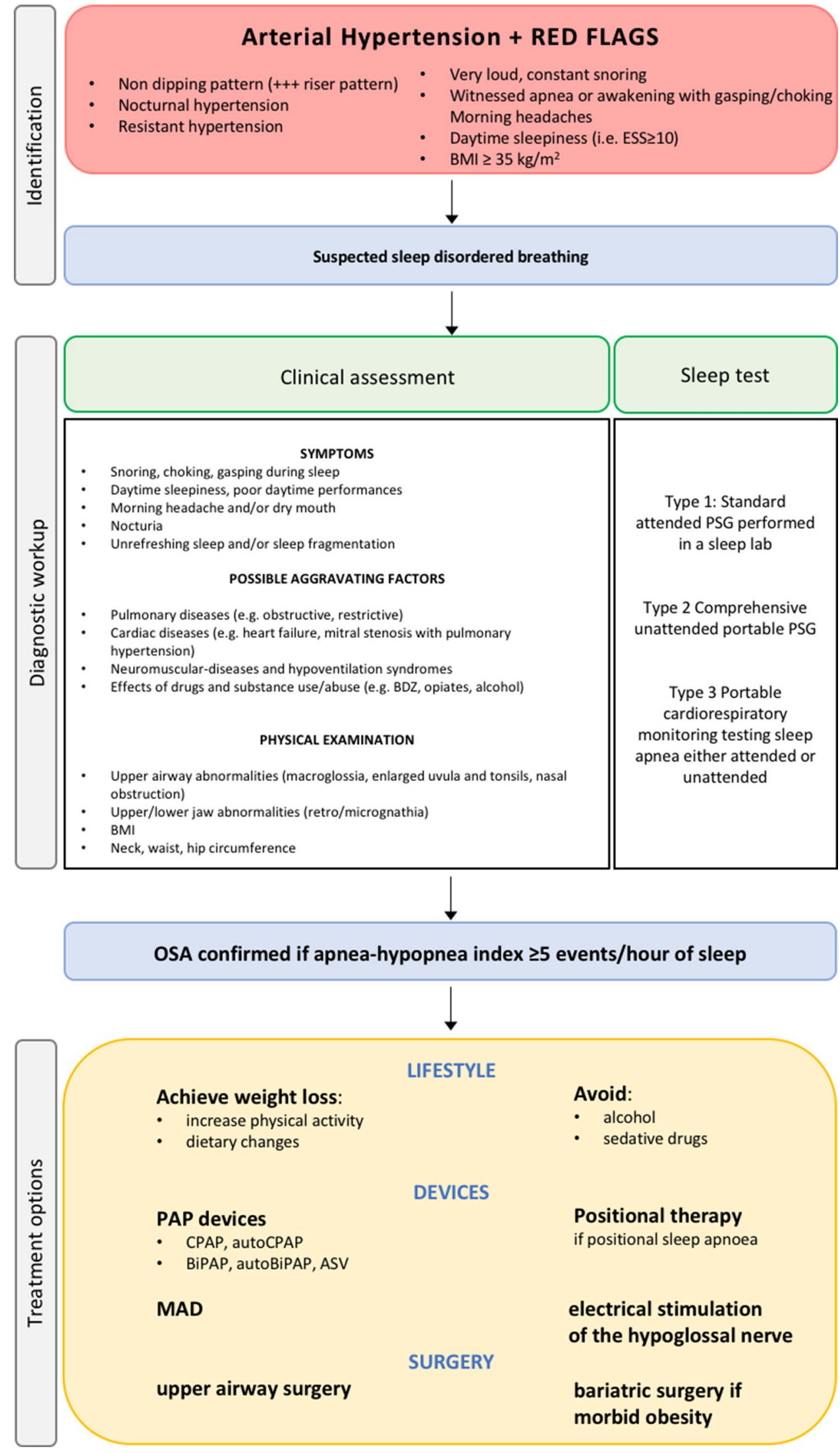


Fig. 4. Diagnostic workup and treatment options for insomnia. See text for details. $O S A$ obstructive sleep apnoea, $B D Z$ benzodiazepines, ESS Epworth sleepiness scale, ISI insomnia severity index, DBAS-16 dysfunctional beliefs and attitudes about sleep

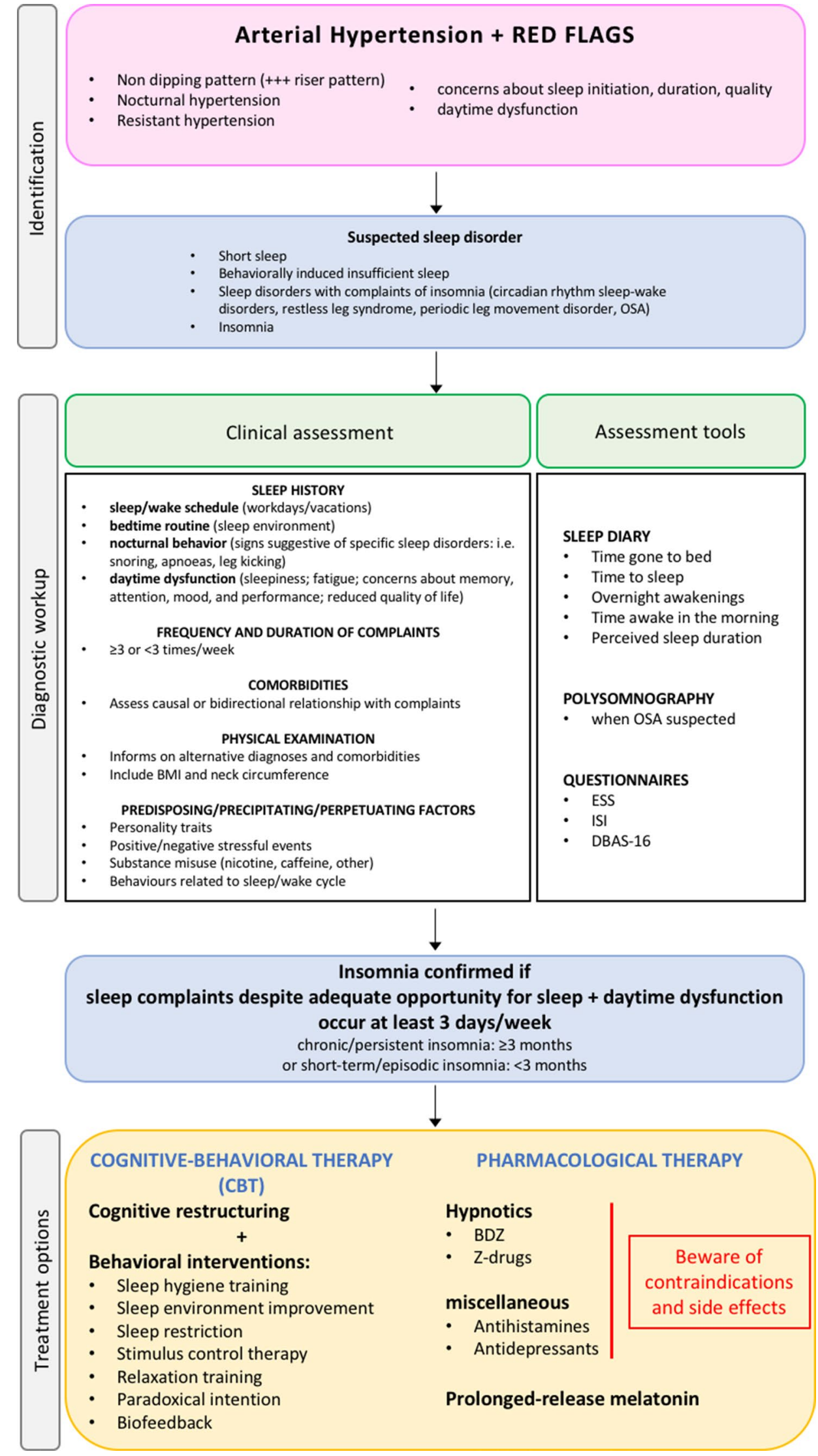


for insomnia in patients over 55 years of age, does not act on GABA receptors [60].

Hypnotics differ mainly in terms of elimination, half-life and principle of action. Some hypnotics (e.g., first-generation benzodiazepines) have greater chances of inducing residual sedation in the morning, especially after prolonged use and/or in the elderly, in which they may also increase the risk of falls, episodes of confusion and impaired cognitive performance.

In recent decades, selective hypnoinducers, so-called Z-drugs (such as zolpidem and zopiclone) have been developed, which can theoretically be taken even in conjunction with an infra-hypnic awakening provided that patients have the chance to sleep for at least $4 \mathrm{~h}$ after administration.

Hypnotics should be used with caution in patients with respiratory failure. In the elderly, any hypnotic, even at reduced doses, can cause restlessness, psychomotor agitation, or exacerbation of states such as delirium and dementia.

Difficulties can be minimised by using the lowest effective dose for brief periods and gradually reducing the dose before stopping the drug.

It should also be recalled that both European guidelines and an Italian consensus indicate $2 \mathrm{mg}$ prolonged-release melatonin as the first therapeutic choice in insomniac subjects over the age of 55 years for up to 13 weeks [17, 60]. In addition to benzodiazepines and hypno-inducing drugs, there are also many other drugs not specifically indicated for insomnia that are used to induce and maintain sleep. Antihistamines such as doxorubicin and diphenhydramine can induce sleep. However, their efficacy is variable; moreover, these drugs can cause adverse effects such as confusion, urinary retention and possible systemic anticholinergic side effects, which are potentially serious in the elderly.

Antidepressants taken at low doses at bedtime (e.g. 5-20 $\mathrm{mg}$ paroxetine, $50 \mathrm{mg}$ trazodone, $75-200 \mathrm{mg}$ trimipramine) may improve sleep. However, they should be used at low doses mainly when standard hypnotics are not tolerated (rare), or in high doses (antidepressants) when concomitant depression of mood is present.

Melatonin is a hormone that is secreted by the pineal gland (and is found naturally in some foods such as oats, almonds and corn, for example). Darkness stimulates secretion, whereas light inhibits it. By binding melatonin receptors in the suprachiasmatic nucleus, melatonin regulates the circadian rhythm and especially the sleep-wake cycle. Exogenous melatonin supplementation may be indicated in various sleep disturbance conditions: jet lag, fragmented sleep or delayed sleep phase syndrome. Oral melatonin can be administered in different dosages and formulations depending on the condition we are treating (typically from 0.5 to 5 $\mathrm{mg}$ before bedtime). The key element is the time of administration, which must be regular and chosen on the basis of the sleep disorder we are dealing with $[61,62]$. The choice of the product to be taken is therefore key, since most of the available melatonin products are "over-the-counter" supplements and sometimes the actual dosage of melatonin they contain is not well regulated.

In Italy there is only one product registered as a prolonged-release melatonin-based drug at a dose of $2 \mathrm{mg}$ [17].

Treatment response can be evaluated with the readministration of questionnaires first collected at baseline for the assessment of insomnia severity (Insomnia Severity Index, ISI [63]) or sleep related beliefs and attitudes in insomnia (Dysfunctional Beliefs and Attitudes about Sleep short version, DBAS-16 [64]) (Figs. 4, 5).

\subsection{The Impact of the Treatment of Sleep Disorders on Blood Pressure}

\subsubsection{Obstructive Sleep Apnoea}

Several studies have shown that treatment of OSA may allow a significant, albeit modest, reduction in BP. In particular, among the treatments specific to OSA, those that have been studied with regard to their effect on BP are CPAP devices and MAD. Recent meta-analyses indicate a significant but small reduction in BP values in OSA patients treated with CPAP [65-67], with more evident effects on patients with resistant hypertension and on overnight $\mathrm{BP}$ values. This is interesting because the role of nocturnal BP in the development of cardiovascular diseases and its association with cardiovascular mortality and morbidity both in hypertensive patients and in the general population has been confirmed in several longitudinal and population studies [68]. In addition to higher baseline BP values, excessive daytime sleepiness and, in particular, increased treatment compliance are also associated with increased positive effects of CPAP treatment on $\mathrm{BP}[69,70]$.

Oral devices are indicated for the treatment of mild to moderate OSA and their effect on BP values is promising, similar to that obtained with CPAP according to some studies. Recently, a meta-analysis [68] comprising data from 68 randomised controlled studies comparing CPAP or MAD with passive or active treatment demonstrated that CPAP was associated with a mean reduction in BP of -2.09 (CI $95 \%-2.78$ to 1.40$) \mathrm{mmHg}$ for systolic pressure and -1.92 (CI 95\% - 2.40 to 1.43) $\mathrm{mmHg}$ for diastolic pressure, while the corresponding values for MAD were -1.27 (CI 95\% -2.34 to -0.20$) \mathrm{mmHg}$ for systolic pressure and -1.11 (CI 95\% - 1.82 to -0.41 ) $\mathrm{mmHg}$ for diastolic pressure. A subgroup analysis showed that patients with higher BP values prior to treatment, patients $<60$ years of age, and patients with higher hypoxic load were more likely to exhibit a more pronounced hypotensive effect in response to CPAP treatment. 
Fig. 5. Common questionnaires for detection and monitoring of insomnia. See text for details
Box 1.

ISI

1. Difficulty falling asleep

2. Difficulty steying asleep

3. Problem waking up too early in the morning

4. How satisfied are you with your current sleep pattern?

5 . To what extent do you consider your sleep problem to interfere with your daily functioning?

6 . How worried are you about your current sleep problem?

7. How noticeable to others do you think your sleep problem is in terms of impairing the quality of your life?

Total scores

$\begin{array}{lllll}0 & 1 & 2 & 3 & 4 \\ 0 & 1 & 2 & 3 & 4 \\ 0 & 1 & 2 & 3 & 4 \\ 0 & 1 & 2 & 3 & 4 \\ 0 & 1 & 2 & 3 & 4\end{array}$

Refer to the PAST MONTH.

Questions 1-3: rate severity ( 0 : none; 1: mild; 2: moderate; 3: severe; 4: very severe).

Question 4: rate satisfaction (0: very satisfied; 1: satisfied; 2: neutral; 3: dissatisfied; 4: very dissatisfied).

Questions 5-7: rate magnitude (0: not at all; 1: a little; 2: somewhat; 3: much; 4: very much).

Scores 0-7 = No clinically significant insomnia

Scores 8-14 = Subthreshold insomnia

Scores 15-21 = Clinical insomnia (moderate severity)

Scores 22-28 = Clinical insomnia (severe)

Box 2.

DBAS-16

1. I need 8 hours of sleep to feel refreshed and function well during the day.

2. When I don't get proper amount of sleep on a given night, I need to catch up on the next day by napping or on the next night by sleeping longer.

3. I am concerned that chronic insomnia may have serious consequences on my physical health.

4. I am worried that I may lose control over my abilities to sleep.

5. After a poor night's sleep, I know that it will interfere with my daily activities on the next day.

6. In order to be alert and function well during the day, I believe I would be better off taking a

sleeping pill rather than having a poor night's sleep.

7. When I feel irritable, depressed, or anxious during the day, it is mostly because I did not sleep well the night before.

8. When I sleep poorly on one night, I know it will disturb my sleep schedule for the whole week.

9. Without an adequate night's sleep, I can hardly function the next day.

10. I can't ever predict whether I'll have a good or poor night's sleep.

11. I have little ability to manage the negative consequences of disturbed sleep.

12. When I feel tired, have no energy, or just seem not to function well during the day, it is generally because I did not sleep well the night before.

13. I believe insomnia is essentially the result of a chemical imbalance.

14. I feel insomnia is ruining my ability to enjoy life and prevents me from doing what I want.

15. Medication is probably the only solution to sleeplessness.

16. I avoid or cancel obligations (social, family) after a poor night's sleep.

For each statement, please indicate to what extent you personally agree or disagree $(0=$ strongly disagree; $10=$ strongly agree).
In terms of medical therapy, the most promising class of antihypertensive drugs capable of reducing both the severity of OSA and BP values are diuretics, particularly mineralocorticoid receptor antagonists, whose beneficial effects in this context are believed to derive from the reduction of peripheral oedema and volume overload [71], and the consequent reduction in the volume shift towards neck and central circulation which occurs when taking the supine position. In addition, other classes of antihypertensive drugs that showed convincing results have been tested. Eskandari 
et al. demonstrated that acetazolamide alone and in combination with CPAP reduced BP values, vascular rigidity and respiratory sleep disorders in patients with OSA and arterial hypertension [72]. Furthermore, in a French study, it was shown that, in patients with OSA, treatment with valsartan resulted in a greater reduction in BP than in CPAP alone, not only during the day but also at night [73].

\subsubsection{Other Sleep Disorders}

With regard to non-respiratory sleep disorders, a systematic review of the effects of prolonged sleep time on cardiometabolic risk factors [74] included only three small-scale studies in which BP was evaluated. None of these reported a significant positive effect. Conversely, a recent study that applied cognitive-behavioural therapy to prolong sleep in "short sleeper" adults and with prehypertension or stage one hypertension found significant reductions in BP with treatment [75]. There is little data on the beneficial effect of the treatment of insomnia and RLS on BP and it is often controversial.

Encouraging data on the possibility of reducing BP in sleep disorders are available for evening administration of prolonged-release melatonin $2 \mathrm{mg}[76,77]$.

In patients with moderate to severe RLS, rotigotine compared to placebo appears to significantly reduce the acute spikes in BP associated with PLMS [78]. Finally, there are few studies on the BP effects of sleep disorder treatment in shift workers. A recent systematic Cochrane review of the pharmacological interventions for sleepiness and sleep disorders caused by shift work highlighted the need for further and better-quality studies on the effects and costs of all pharmacological agents that induce sleep or promote alertness in shift workers, both with and without a diagnosis of shift work sleep disorder [79].

\section{Conclusions}

This document by the Italian Society of Hypertension on qualitative and/or quantitative sleep disorders aimed to define a shared approach to a condition with a significant negative impact on overall cardiometabolic risk and related fatal/non-fatal events. At present, in fact, several open questions exist concerning the diagnosis, management and therapy of hypertensive patients with sleep disorders. Although guidelines on arterial hypertension recommend investigating sleep quality/quantity as well as the presence of OSA, and specifically treating the latter in the hypertensive patient, several relevant aspects remain undetermined. These include the severity of respiratory disorder and BP threshold for indications for specific therapies; the most appropriate BP measurement tools in this context; the predictive factors of
BP response, including the effects of antihypertensive drugs and lifestyle/weight interventions; and the management of insomnia in patients with obstructive apnoea [13, 27].

In addition to the evidence supporting the benefit of CPAP in terms of BP reduction in hypertensive patients with OSA, there is no data supporting that this measure reduces the risk of major fatal/nonfatal events, independent of apnoea severity, duration of follow-up, or treatment adherence [80]. At the same time, however, a certain degree of reversibility in the cardiovascular risk profile following treatment appears to be achievable [27]. It is likely that the magnitude of this benefit might depend on concomitant nonmodifiable factors, such as age or pre-existing irreversible organ damage, which would therefore affect the indications for specific treatments.

Consideration is also needed as to whether BP increases-both in terms of mean BP values and BP variability - that may potentially be observed during the use of CPAP depend on technical factors-such as air leaks from the mask or excessive positive pressure. These changes can be responsible for night-time awakening or represent a source of considerable discomfort for the patient, leading to discontinuation of treatment [27, 81].

Therefore, the appropriate management of patients with OSA and hypertension should be multifactorial, including: optimisation of type, number and doses of antihypertensive drugs; improvement of adherence to pharmacological and non-pharmacological treatments; implementation of lifestyle changes based on the overall cardiovascular risk; and identification and appropriate treatment of insomnia. Care to start treatment as early as possible in the clinical history of OSA, before structural cardiovascular changes might occur, also needs to be implemented.

Further research is needed to better clarify the relationship between hypertension and sleep disorders, in the light of the many possible confounding elements. Similarly, dedicated studies that objectively assess the duration, quality, timing and regularity of sleep in relation to incident hypertension, identify the underlying genetic and molecular events, and define the role of more or less complex interventions (surgical procedures, use of intraoral devices, or selective nerve stimulation) in populations with different disease severity or baseline cardiovascular risk may clarify the significance of the observed associations. Pending these developments, it is mandatory to reduce the "time to diagnosis" of sleep disorders and relevant comorbidities in the hypertensive patient, especially if resistant to therapy and/ or at high cardiovascular risk, and to improve the available therapeutic strategies and adherence to them. In this context, it is worth it to investigate more thoroughly whether restoring the melatoninergic system through prolonged-release melatonin at suitable doses [17] may represent, along with lifestyle changes, a widely applicable option. To date, the 
triad "lifestyle changes + medications + possible device" stands as the only evidence-based approach.

Acknowledgements None. SIIA Young Researchers Study Group: Rita Del Pinto; President of SIIA: Guido Grassi; Past President of SIIA: Claudio Ferri; The Italian Society of Hypertension Board: Prof. Guido Grassi, Prof. Claudio Ferri, Prof. Maria Lorenza Muiesan, Prof. Massimo Salvetti, Prof. Leonardo Sechi, Prof. Arrigo F. G. Cicero, Prof. Guido Iaccarino, Prof. Pietro Minuz, Prof. Paolo Mulatero, Prof. Giuseppe Mulè, Dott. Giacomo Pucci, Prof. Carmine Savoia. Scientific Committee: Prof. Claudio Borghi, Prof. Claudio Ferri, Prof. Enrico Agabiti Rosei, Prof. Francesco Cipollone, Prof. Giovambattista Desideri, Prof. Agostino Virdis, Prof. Massimo Volpe.

\section{Declarations}

Funding Open access funding provided by Università degli Studi dell'Aquila within the CRUI-CARE Agreement.

\section{Conflict of interest The authors declare no conflicts of interest}

Ethics approval This is a consensus document based on a review of the existing evidence, and the local Research Ethics Committee has confirmed that no ethical approval is required.

Consent to participate and Consent for publication As a consensus document based on a review of the existing evidence, it did not enroll individual participants, and consent to participate and for publications were therefore not required.

Availability of data and material Not applicable.

Code availability Not applicable.

Open Access This article is licensed under a Creative Commons Attribution-NonCommercial 4.0 International License, which permits any non-commercial use, sharing, adaptation, distribution and reproduction in any medium or format, as long as you give appropriate credit to the original author(s) and the source, provide a link to the Creative Commons licence, and indicate if changes were made. The images or other third party material in this article are included in the article's Creative Commons licence, unless indicated otherwise in a credit line to the material. If material is not included in the article's Creative Commons licence and your intended use is not permitted by statutory regulation or exceeds the permitted use, you will need to obtain permission directly from the copyright holder. To view a copy of this licence, visit http://creativecommons.org/licenses/by-nc/4.0/.

\section{References}

1. Adam K, Oswald I. Protein synthesis, bodily renewal and the sleep-wake cycle. Clin Sci. 1983;65:561-7.

2. Vgontzas AN, Mastorakos G, Bixler EO, Kales A, Gold PW, Chrousos GP. Sleep deprivation effects on the activity of the hypothalamic-pituitary-adrenal and growth axes: potential clinical implications. Clin Endocrinol. 1999;51:205-15.

3. Tobaldini E, Fiorelli EM, Solbiati M, Costantino G, Nobili L, Montano N. Short sleep duration and cardiometabolic risk: from pathophysiology to clinical evidence. Nat Rev Cardiol. 2019;16:213-24.
4. Williams B, Mancia G, Spiering W, Agabiti Rosei E, Azizi M, Burnier M, et al. 2018 ESC/ESH Guidelines for the management of arterial hypertension. Eur Heart J. 2018;39:3021-104.

5. Parati G, Lombardi C, Hedner J, Bonsignore MR, Grote L, Tkacova R, et al. Position paper on the management of patients with obstructive sleep apnea and hypertension: joint recommendations by the European Society of Hypertension, by the European Respiratory Society and by the members of European COST (COoperation in Scientific and Technological research) ACTION B26 on obstructive sleep apnea. J Hypertens. 2012;30:633-46.

6. Cappuccio FP, Stranges S, Kandala N-B, Miller MA, Taggart FM, Kumari M, et al. Gender-specific associations of short sleep duration with prevalent and incident hypertension: the Whitehall II Study. Hypertension. 2007;50:693-700.

7. Aggarwal B, Makarem N, Shah R, Emin M, Wei Y, St-Onge M-P, et al. Effects of inadequate sleep on blood pressure and endothelial inflammation in women: findings from the American Heart Association Go Red for Women Strategically Focused Research Network. J Am Heart Assoc. 2018. https://doi.org/10.1161/ JAHA.118.008590.

8. Möller-Levet CS, Archer SN, Bucca G, Laing EE, Slak A, Kabiljo $\mathrm{R}$, et al. Effects of insufficient sleep on circadian rhythmicity and expression amplitude of the human blood transcriptome. Proc Natl Acad Sci USA. 2013;110:E1132-41.

9. Kim JH, Hayek SS, Ko Y-A, Liu C, Samman Tahhan A, Ali S, et al. Sleep duration and mortality in patients with coronary artery disease. Am J Cardiol. 2019;123:874-81.

10. Xiao Q, Keadle SK, Hollenbeck AR, Matthews CE. Sleep duration and total and cause-specific mortality in a large US cohort: interrelationships with physical activity, sedentary behavior, and body mass index. Am J Epidemiol. 2014;180:997-1006.

11. Cappuccio FP, D'Elia L, Strazzullo P, Miller MA. Sleep duration and all-cause mortality: a systematic review and meta-analysis of prospective studies. Sleep. 2010;33:585-92.

12. Luyster FS, Buysse DJ, Strollo PJ Jr. Comorbid insomnia and obstructive sleep apnea: challenges for clinical practice and research. J Clin Sleep Med. 2010;6:196-204.

13. Ong JC, Crawford MR. Insomnia and obstructive sleep apnea. Sleep Med Clin. 2013;8:389-98.

14. Janssen HCJP, Venekamp LN, Peeters GAM, Pijpers A, Pevernagie DAA. Management of insomnia in sleep disordered breathing. Eur Respir Rev. 2019. https://doi.org/10.1183/16000 617.0080-2019.

15. Mason M, Cates CJ, Smith I. Effects of opioid, hypnotic and sedating medications on sleep-disordered breathing in adults with obstructive sleep apnoea. Cochrane Database Syst Rev. 2015; CD011090.

16. Forman JP, Curhan GC, Schernhammer ES. Urinary melatonin and risk of incident hypertension among young women. J Hypertens. 2010;28:446-51.

17. Palagini L, Manni R, Aguglia E, Amore M, Brugnoli R, Girardi $\mathrm{P}$, et al. Expert opinions and consensus recommendations for the evaluation and management of insomnia in clinical practice: Joint Statements of Five Italian Scientific Societies. Front Psychiatry. 2020;11:558.

18. AASM Scoring Manual-American Academy of Sleep Medicine. [cited 17 Sep 2020]. https://aasm.org/clinical-resources/scoringmanual/.

19. (jssr): SCCOFTJSOFSRS, SLEEP COMPUTING COMMITTEE OF THE JAPANESE SOCIETY OF SLEEP RESEARCH SOCIETY (JSSR): Hori T, Sugita Y, Koga E, Shirakawa S, Proposed supplements and amendments to "A Manual of Standardized Terminology, Techniques and Scoring System for Sleep Stages of Human Subjects", the Rechtschaffen \& Kales, , et al. standard. Psychiatry Clin Neurosci. 1968;2001:305-10. https://doi.org/10. 1046/j.1440-1819.2001.00810.x. 
20. Terjung R. Arterial baroreflexes in humans. Comprehensive physiology. Hoboken: Wiley; 2011. p. 52.

21. Ito E, Inoue Y. The International Classification of Sleep Disorders, third edition. American Academy of Sleep Medicine. Includes bibliographies and index. Nihon Rinsho. 2015;73:916-23.

22. Baglioni C, Battagliese G, Feige B, Spiegelhalder K, Nissen C, Voderholzer $\mathrm{U}$, et al. Insomnia as a predictor of depression: a meta-analytic evaluation of longitudinal epidemiological studies. J Affect Disord. 2011. https://doi.org/10.1016/j.jad.2011.01.011.

23. Hertenstein E, Feige B, Gmeiner T, Kienzler C, Spiegelhalder K, Johann A, et al. Insomnia as a predictor of mental disorders: a systematic review and meta-analysis. Sleep Med Rev. 2019;43:96-105.

24. Pigeon WR, Pinquart M, Conner K. Meta-analysis of sleep disturbance and suicidal thoughts and behaviors. J Clin Psychiatry. 2012. https://doi.org/10.4088/jcp.11r07586.

25. Sofi F, Cesari F, Casini A, Macchi C, Abbate R, Gensini GF. Insomnia and risk of cardiovascular disease: a meta-analysis. Eur J Prev Cardiol. 2014. https://doi.org/10.1177/204748731246002 0 .

26. Lanfranchi PA, Pennestri M-H, Fradette L, Dumont M, Morin $\mathrm{CM}$, Montplaisir J. Night-time blood pressure in normotensive subjects with chronic insomnia: implications for cardiovascular risk. Sleep. 2009;32:760-6.

27. Lombardi C, Pengo MF, Parati G. Systemic hypertension in obstructive sleep apnea. J Thorac Dis. 2018;10:S4231-43.

28. Winkelman JW, Blackwell T, Stone K, Ancoli-Israel S, Redline S. Associations of incident cardiovascular events with restless legs syndrome and periodic leg movements of sleep in older men, for the outcomes of sleep disorders in Older Men Study (MrOS Sleep Study). Sleep. 2017. https://doi.org/10.1093/sleep/zsx023.

29. Sieminski M, Partinen M. Nocturnal systolic blood pressure is increased in restless legs syndrome. Sleep Breath. 2016;20:1013-9.

30. Ohayon MM, Smirne S. Prevalence and consequences of insomnia disorders in the general population of Italy. Sleep Med. 2002;3:115-20.

31. St-Onge M-P, Grandner MA, Brown D, Conroy MB, Jean-Louis G, Coons M, et al. Sleep duration and quality: impact on lifestyle behaviors and cardiometabolic health: a scientific statement from the American Heart Association. Circulation. 2016;134:e367-86.

32. Cappuccio FP, D'Elia L, Strazzullo P, Miller MA. Quantity and quality of sleep and incidence of type 2 diabetes: a systematic review and meta-analysis. Diabetes Care. 2010;33:414-20.

33. Guo X, Zheng L, Wang J, Zhang X, Zhang X, Li J, et al. Epidemiological evidence for the link between sleep duration and high blood pressure: a systematic review and meta-analysis. Sleep Med. 2013;14:324-32.

34. Jiang W, Hu C, Li F, Hua X, Zhang X. Association between sleep duration and high blood pressure in adolescents: a systematic review and meta-analysis. Ann Hum Biol. 2018;45:457-62.

35. Krittanawong C, Kumar A, Wang Z, Jneid H, Baber U, Mehran $\mathrm{R}$, et al. Sleep duration and cardiovascular health in a representative community population (from NHANES, 2005 to 2016). Am J Cardiol. 2020;127:149-55.

36. Cappuccio FP, Cooper D, D’Elia L, Strazzullo P, Miller MA. Sleep duration predicts cardiovascular outcomes: a systematic review and meta-analysis of prospective studies. Eur Heart J. 2011;32:1484-92.

37. Daghlas I, Dashti HS, Lane J, Aragam KG, Rutter MK, Saxena R, et al. Sleep duration and myocardial infarction. J Am Coll Cardiol. 2019;74:1304-14.

38. Chokesuwattanaskul R, Thongprayoon $\mathrm{C}$, Sharma K, Congrete S, Tanawuttiwat T, Cheungpasitporn W. Associations of sleep quality with incident atrial fibrillation: a meta-analysis. Intern Med J. 2018;48:964-72.

39. Morovatdar N, Ebrahimi N, Rezaee R, Poorzand H, Bayat Tork MA, Sahebkar A. Sleep duration and risk of atrial fibrillation: a systematic review. J Atr Fibrillation. 2019;11:2132.

40. Christensen MA, Dixit S, Dewland TA, Whitman IR, Nah G, Vittinghoff $\mathrm{E}$, et al. Sleep characteristics that predict atrial fibrillation. Heart Rhythm. 2018;15:1289-95.

41. Ramos AR, Weng J, Wallace DM, Petrov MR, Wohlgemuth WK, Sotres-Alvarez D, et al. Sleep patterns and hypertension using actigraphy in the hispanic community health study/study of Latinos. Chest. 2018;153:87-93.

42. Heinzer R, Vat S, Marques-Vidal P, Marti-Soler H, Andries D, Tobback N, et al. Prevalence of sleep-disordered breathing in the general population: the HypnoLaus study. Lancet Respir Med. 2015;3:310-8.

43. Bouloukaki I, Grote L, McNicholas WT, Hedner J, Verbraecken J, Parati G, et al. Mild obstructive sleep apnea increases hypertension risk, challenging traditional severity classification. J Clin Sleep Med. 2020;16:889-98.

44. Gündüz C, Basoglu OK, Hedner J, Zou D, Bonsignore MR, Hein $\mathrm{H}$, et al. Obstructive sleep apnoea independently predicts lipid levels: data from the European Sleep Apnea Database. Respirology. 2018;23:1180-9.

45. Ayas NT, Hirsch AAJ, Laher I, Bradley TD, Malhotra A, Polotsky VY, et al. New frontiers in obstructive sleep apnoea. Clin Sci. 2014;127:209-16.

46. Azarbarzin A, Sands SA, Stone KL, Taranto-Montemurro L, Messineo L, Terrill PI, et al. The hypoxic burden of sleep apnoea predicts cardiovascular disease-related mortality: the Osteoporotic Fractures in Men Study and the Sleep Heart Health Study. Eur Heart J. 2019;40:1149-57.

47. Gottlieb DJ, Yenokyan G, Newman AB, O'Connor GT, Punjabi NM, Quan SF, et al. Prospective study of obstructive sleep apnea and incident coronary heart disease and heart failure: the sleep heart health study. Circulation. 2010;122:352-60.

48. Chung F, Yegneswaran B, Liao P, Chung SA, Vairavanathan S, Islam S, et al. STOP questionnaire: a tool to screen patients for obstructive sleep apnea. Anesthesiology. 2008;108:812-21.

49. Farney RJ, Walker BS, Farney RM, Snow GL, Walker JM. The STOP-Bang equivalent model and prediction of severity of obstructive sleep apnea: relation to polysomnographic measurements of the apnea/hypopnea index. J Clin Sleep Med. 2011;7:459-65.

50. Johns MW. A new method for measuring daytime sleepiness: the Epworth sleepiness scale. Sleep. 1991;14:540-5.

51. Netzer NC, Stoohs RA, Netzer CM, Clark K, Strohl KP. Using the Berlin Questionnaire to identify patients at risk for the sleep apnea syndrome. Ann Intern Med. 1999;131:485-91.

52. Takegami M, Hayashino Y, Chin K, Sokejima S, Kadotani H, Akashiba T, et al. Simple four-variable screening tool for identification of patients with sleep-disordered breathing. Sleep. 2009;32:939-48.

53. Guo Q, Song W-D, Li W, Zeng C, Li Y-H, Mo J-M, et al. Weighted Epworth sleepiness scale predicted the apnea-hypopnea index better. Respir Res. 2020;21:147.

54. Haba-Rubio J, Marti-Soler H, Tobback N, Andries D, MarquesVidal P, Vollenweider P, et al. Clinical significance of periodic limb movements during sleep: the HypnoLaus study. Sleep Med. 2018;41:45-50.

55. Gottlieb DJ, Somers VK, Punjabi NM, Winkelman JW. Restless legs syndrome and cardiovascular disease: a research roadmap. Sleep Med. 2017;31:10-7.

56. Bøggild H, Knutsson A. Shift work, risk factors and cardiovascular disease. Scand J Work Environ Health. 1999;25:85-99. 
57. Torquati L, Mielke GI, Brown WJ, Kolbe-Alexander T. Shift work and the risk of cardiovascular disease. A systematic review and meta-analysis including dose-response relationship. Scand J Work Environ Health. 2018;44:229-38.

58. Malhotra A, Mesarwi O, Pepin J-L, Owens RL. Endotypes and phenotypes in obstructive sleep apnea. Curr Opin Pulm Med. 2020. https://doi.org/10.1097/MCP.0000000000000724.

59. Krystal AD, Prather AA, Ashbrook LH. The assessment and management of insomnia: an update. World Psychiatry. 2019;18(3):337-52. https://doi.org/10.1002/wps.20674.

60. Wilson S, Anderson K, Baldwin D, Dijk D-J, Espie A, Espie C, et al. British Association for Psychopharmacology consensus statement on evidence-based treatment of insomnia, parasomnias and circadian rhythm disorders: an update. J Psychopharmacol. 2019;33:923-47.

61. Riemann D, Baglioni C, Bassetti C, Bjorvatn B, Dolenc Groselj L, Ellis JG, et al. European guideline for the diagnosis and treatment of insomnia. J Sleep Res. 2017;26:675-700.

62. Sateia MJ, Buysse DJ, Krystal AD, Neubauer DN, Heald JL. Clinical practice guideline for the pharmacologic treatment of chronic insomnia in adults: an American Academy of Sleep Medicine Clinical Practice Guideline. J Clin Sleep Med. 2017;13:307-49.

63. Morin CM, Belleville G, Bélanger L, Ivers H. The Insomnia Severity Index: psychometric indicators to detect insomnia cases and evaluate treatment response. Sleep. 2011;34:601-8.

64. Morin CM, Vallières A, Ivers H. Dysfunctional beliefs and attitudes about sleep (DBAS): validation of a brief version (DBAS16). Sleep. 2007;30:1547-54.

65. Hu X, Fan J, Chen S, Yin Y, Zrenner B. The role of continuous positive airway pressure in blood pressure control for patients with obstructive sleep apnea and hypertension: a meta-analysis of randomized controlled trials. J Clin Hypertens. 2015;17:215-22.

66. Iftikhar IH, Valentine CW, Bittencourt LRA, Cohen DL, Fedson AC, Gíslason T, et al. Effects of continuous positive airway pressure on blood pressure in patients with resistant hypertension and obstructive sleep apnea: a meta-analysis. J Hypertens. 2014;32:2341-50.

67. Schein ASO, Kerkhoff AC, Coronel CC, Plentz RDM, Sbruzzi G. Continuous positive airway pressure reduces blood pressure in patients with obstructive sleep apnea; a systematic review and meta-analysis with 1000 patients. J Hypertens. 2014;32:1762-73.

68. Pengo MF, Soranna D, Giontella A, Perger E, Mattaliano P, Schwarz EI, et al. Obstructive sleep apnoea treatment and blood pressure: which phenotypes predict a response? A systematic review and meta-analysis. Eur Respir J. 2020. https://doi. org/10.1183/13993003.01945-2019.

69. Torres G, Sánchez-de-la-Torre M, Barbé F. Relationship between OSA and hypertension. Chest. 2015;148:824-32.
70. Parati G, Pengo MF, Lombardi C. Obstructive sleep apnea and hypertension: why treatment does not consistently improve blood pressure. Curr Hypertens Rep. 2019;21:30.

71. Gaddam K, Pimenta E, Thomas SJ, Cofield SS, Oparil S, Harding SM, et al. Spironolactone reduces severity of obstructive sleep apnoea in patients with resistant hypertension: a preliminary report. J Hum Hypertens. 2010;24:532-7.

72. Eskandari D, Zou D, Grote L, Hoff E, Hedner J. Acetazolamide reduces blood pressure and sleep-disordered breathing in patients with hypertension and obstructive sleep apnea: a randomized controlled trial. J Clin Sleep Med. 2018;14:309-17.

73. Pépin J-L, Tamisier R, Barone-Rochette G, Launois SH, Lévy P, Baguet J-P. Comparison of continuous positive airway pressure and valsartan in hypertensive patients with sleep apnea. Am J Respir Crit Care Med. 2010;182:954-60.

74. Henst RHP, Pienaar PR, Roden LC, Rae DE. The effects of sleep extension on cardiometabolic risk factors: a systematic review. J Sleep Res. 2019;28:e12865.

75. Baron KG, Duffecy J, Richardson D, Avery E, Rothschild S, Lane J. Technology assisted behavior intervention to extend sleep among adults with short sleep duration and prehypertension/stage 1 hypertension: a randomized pilot feasibility study. J Clin Sleep Med. 2019;15:1587-97.

76. Pourhanifeh MH, Dehdashtian E, Hosseinzadeh A, Sezavar SH, Mehrzadi S. Clinical application of melatonin in the treatment of cardiovascular diseases: current evidence and new insights into the cardioprotective and cardiotherapeutic properties. Cardiovasc Drugs Ther. 2020. https://doi.org/10.1007/s10557-020-07052-3.

77. Grossman E, Laudon M, Zisapel N. Effect of melatonin on nocturnal blood pressure: meta-analysis of randomized controlled trials. Vasc Health Risk Manag. 2011;7:577-84.

78. Bauer A, Cassel W, Benes H, Kesper K, Rye D, Sica D, et al. Rotigotine's effect on PLM-associated blood pressure elevations in restless legs syndrome: an RCT. Neurology. 2016;86:1785-93.

79. Liira J, Verbeek JH, Costa G, Driscoll TR, Sallinen M, Isotalo LK, et al. Pharmacological interventions for sleepiness and sleep disturbances caused by shift work. Cochrane Database Syst Rev. 2014; CD009776.

80. Yu J, Zhou Z, McEvoy RD, Anderson CS, Rodgers A, Perkovic V, et al. Association of positive airway pressure with cardiovascular events and death in adults with sleep apnea: a systematic review and meta-analysis. JAMA. 2017;318:156-66.

81. Ratneswaran C, Pengo MF, Xiao S, Luo Y, Rossi GP, Polkey MI, et al. The acute effect of continuous positive airway pressure titration on blood pressure in awake overweight/obese patients with obstructive sleep apnoea. Blood Press. 2018;27:206-14. 